\title{
The G Protein-Coupled Receptor, VPAC1, is The Principle Driver for VIP Deficiency-Induced Alterations of Gut Microbiota in C57BL/6 Mice.
}

\author{
Bains Manpreet \\ North Dakota State University \\ James Waschek \\ University of California, Los Angeles \\ Aaron Ericsson \\ University of Missouri \\ Glenn Dorsam ( $\nabla$ glenn.dorsam@ndsu.edu ) \\ North Dakota State University
}

\section{Research Article}

Keywords: VIP, microbiota, signaling, VPAC1

Posted Date: January 28th, 2021

DOI: https://doi.org/10.21203/rs.3.rs-150055/v1

License: (c) (i) This work is licensed under a Creative Commons Attribution 4.0 International License.

Read Full License

Version of Record: A version of this preprint was published at Gastro Hep Advances on November 18th, 2021. See the published version at https://doi.org/10.1016/j.gastha.2021.11.005. 


\section{Abstract}

Previous studies in our lab linked vasoactive intestinal peptide (VIP) signaling to gut microbiota stability. Although VIP-deficient mice demonstrated a gut microbiota dysbiosis characterized by a reduction in both the Firmicutes:Bacteroidetes phyla ratio and a-diversity, the mechanism for this observation was not known. To this end, we conducted a similar study analyzing fecal samples from VIP receptor-deficient mice, known as VPAC1 and VPAC2, by 16S rRNA sequencing. Samples from male and female wildtype $(W T, n=30)$, heterozygous (HET, $n=31)$ and homozygous mutant $(K O, n=32)$ mice were analyzed. Our data revealed that genetic deletion of either receptor resulted in statistically significant changes in fecal $\beta$ diversity, with VPAC1 KO samples also demonstrating loss in bacterial a-diversity. Comparing 94 statistically altered OTUs from VPAC1 samples, more than $62 \%$ were similar to those found in VIP KO samples. We conclude that VIP $\rightarrow$ VPAC1 signaling is a major driving force regulating intestinal homeostasis and a stable gut microbiota.

\section{Introduction}

Vasoactive intestinal peptide (VIP) is a 28 amino acid neuropeptide with a broad expression profile regulating diurnal feeding behavior and taste-perception ${ }^{1}$, metabolism ${ }^{2}$ and immunity ${ }^{3}$. VIP is delivered by the peripheral nervous system to numerous organs, including the mucosa-associated lymphoid tissues of the pulmonary and gastrointestinal tract (GIT) ${ }^{4}$. VIP signals with high affinity $\left(K_{d}=1 n M\right)$ through at least two endogenous $G$ protein-coupled receptors (GPCR), called vasoactive intestinal peptide/pituitary adenylyl cyclase activating polypeptide (VPAC) 1 and VPAC2 ${ }^{5,6}$. VIP signaling is responsible for numerous biological effects within the GIT, including digestive enzyme secretion ${ }^{7}$, ion homeostasis ${ }^{8,9}$, relaxation phase of peristalsis ${ }^{10,11}$, mucus secretion ${ }^{12}$, mucosal immunity ${ }^{13}$, intestinal epithelial cell homeostasis (e.g. Goblet cells) and barrier integrity ${ }^{14}$. Importantly, a link between VIP deficiency and reduced expression of a key transcriptional master regulator for intestinal barrier integrity, function and homeostasis, called caudal type homeobox 2 (CDX-2), highlights the profound signaling influence of VIP on homeostasis within the GIT. Moreover, VIP deficient mice have significant morphological abnormalities, including shorter crypt and intestinal lengths, and thickening of smooth muscle tissue surrounding the small intestines ${ }^{12,14}$. Collectively, these data support that VIP plays a central role in GIT function and homeostasis.

It is now well-established that a stable GIT environment is also important for the colonization and maintenance of the gut microbiota ${ }^{15}$. This "invisible metabolic organ" consists of trillions of microorganisms consisting of bacteria, fungi, archaea, eukaryotic parasites and viruses that play a vital role in host physiology, metabolism and immunity [reviewed in ${ }^{16}$ ]. There are many factors that influence the composition of the gut bacterial component of the microbiota (referred to as the gut microbiota from herein), including the mode of child birth ${ }^{17}$, breast feeding ${ }^{18}$, host genetics ${ }^{19,20}$, diet ${ }^{21,22}$ and infection 23 . Diet and infection have been shown to have rapid, but reversible changes in microbiota composition. Support for host genetics influencing the gut microbiota composition comes from a study demonstrating 
that fecal transplants from a donor species to a different, recipient species results in microbiota structures reverting back to that of the recipient host species ${ }^{24}$. Goodrich et al. further contributed evidence for host genetics using a cohort of identical twins to show that nearly $10 \%$ of gut microbiota taxa are heritable, temporally stable and associated with host gene loci involved in diet-sensing, metabolism and immune defense ${ }^{25}$.

Recently, our research group reported that VIP-deficient mice are associated with an altered gut microbiota ecology compared to wild-type (WT) littermates as assessed by culture-independent, 16S rRNA amplicon sequencing ${ }^{19}$. Although this report was the first demonstration that VIP deficiency is associated with changes in the gut microbiota composition, the identity of the specific VIP receptor driving these changes in the gut microbiota remains unknown. Both VIP GPCRs are expressed in the GIT. For example, VPAC1 is localized to the luminal surfaces of intestinal epithelial cells (IECs) ${ }^{5}$. In VIP deficient mice, a lack of VIPàVPAC1 signaling in IECs could explain the observed reduction in MUC2 secretion, the major protein component of mucus ${ }^{26}$. Indeed, both VIP and MUC2 knockout mice possess altered gut microbiota compositional changes, including a depletion of the butyrate-producing Rumminococcaceae lineage ${ }^{19,27}$. On the other hand, VPAC2 is expressed predominantly in smooth muscle cells surrounding the small and large intestines suggesting its involvement in peristalsis ${ }^{28}$, and altered food transit times through the GIT have been found to cause gut microbiota compositional changes ${ }^{29}$. VPAC1 and VPAC2 mRNA are both expressed in innate lymphoid-like 2 cells (ILC2) and VPAC2 mRNA is expressed in CCR6 ${ }^{+}$ILC3 within the intestinal mucosa ${ }^{13,30}$. Moreover, VPAC2 expression in the suprachiasmatic nucleus of the brain has been shown to be essential for VIP-induced diurnal cycling and circadian clock gene synchronization ${ }^{31}$. In addition to diurnal ebbs and wanes of gut microbiota abundance ${ }^{32}$, gut bacterial structures are altered in mouse knockouts for circadian rhythm clock genes and in chickens experiencing altered photoperiods ${ }^{33,34}$ ). Based on this knowledgebase, we hypothesized that VIP receptor deficiency for either receptor would be associated with changes in gut bacterial ecology.

In this report, we show that genetic deletion of VPAC1 in homozygous mutant (KO) samples was associated with substantial changes in the gut microbiota composition compared to VPAC1 heterozygous (HET) or VPAC1 wild type (WT) littermates, irrespective of sex. Interestingly, out of the 94 differentially abundant OTUs in VPAC1 KO samples, there was agreement $(\approx 63 \%)$ with statistically altered OTUs reported for VIP KO samples ${ }^{19}$. VPAC2 deficiency also resulted in differences compared to littermate controls, although the difference was much more subtle relative to the differences found in VPAC1-deficient mice. In total, these data strongly suggest that signaling through both VIP receptors can affect the gut microbiota ecology to different extents and via different, currently unknown, mechanisms. In addition, the VIP $\rightarrow$ VPAC1 signaling axis would appear to provide a more robust and common regulatory influence on the maintenance of the gut microbiota, highlighting this neuropeptide's importance on GIT environmental homeostasis. 


\section{Results}

Sequencing of $16 \mathrm{~S}$ rRNA amplicon libraries generated from a total of 93 mice resulted in a total of $10,076,065$ high-quality sequence reads, and an average of 108,345 reads per sample. The coverage for each specific group is provided in Table 1. To account for the differential coverage between groups, all data were subsampled to a uniform number of reads equivalent to one less than the lowest coverage obtained for any sample (41,866 reads).

Initial comparisons of community richness (Figure 1A) and a-diversity (Figure 1B) in each group revealed a significant reduction in both measures in samples from VPAC1-deficient (KO) mice, relative to wild-type (WT) and heterozygous (HET) littermates. Two-factor ANOVA to identify associations between richness and host sex or genotype within the VPAC1 colony indicated that samples from the VPAC1 KO mice had significantly reduced richness $(p \leq 0.001)$ relative to both WT and HET mice. In contrast, no genotypedependent differences in richness were detected in the VPAC2 colony. No sex-dependent differences were detected in any genotype, or within either colony. Statistical comparison of the Shannon diversity indices revealed an identical pattern. Thus, dysfunctional VPAC1 signaling was associated with a significantly decreased richness and a-diversity, while loss of VPAC2 signaling had no apparent effect.

Resolved to the taxonomic level of phylum, samples from VPAC1-deficient mice demonstrated a significantly greater relative abundance of Proteobacteria and Verrucomicrobia compared to WT and HET littermates, and a decreased relative abundance of Bacteroidetes and Deferribacteres (Figure 2A). There were no clear differences between genotypes within the VPAC2 colony. At the level of Operational Taxonomic Unit (OTU), differences in the gut microbiota (GM) of VPAC1 KO mice became more apparent, with six genera (Bacteroides, Parabacteroides, Lactobacillus, Parasutterella, Escherichia, and Akkermansia) emerging as dominant OTUs comprising a mean ( \pm SD) of $43.6 \%( \pm 12.6 \%)$ of the total DNA in samples from VPAC1-deficient mice (Figure 2B). Curiously, five out of the six dominant genera were Gram-negative bacteria, with Lactobacillus the only enriched Gram-positive genera. To test for genotype-dependent differences in the relative abundance of all detected OTUs, we performed nonparametric Kruskal-Wallis ANOVA on ranks of all OTUs within the VPAC1 colony, using a stringent $p$ value of 0.001 as a cut-off and with rigorous corrections for multiple testing to control the false discovery rate (FDR). Despite this highly conservative approach, we still detected significant differences in the relative abundance of 94 OTUs (Table 2), the majority of which were present at decreased relative abundance in VPAC1 KO mice compared to WT or HET littermates. Those OTUs detected at greater relative abundance in VPAC1 KO mice, included multiple members of the Lactobacillaceae and Peptostreptococcaceae, while taxa at reduced relative abundance in VPAC1 $\mathrm{KO}$ mice included numerous members of the commensal families Lachnospiraceae and Ruminococcaceae. To identify OTUs that were unique to samples from mice expressing VPAC1 (i.e., present in WT and/or HET but completely absent in KO mice) or mice lacking VPAC1 (i.e., present in KO but completely absent in WT and HET mice), a Venn diagram was constructed

(Figure 3A). OTUs detected in only WT or HET mouse (Figures 3B and 3C, respectively) represent taxa that were completely lost (or below the limit of detection) in the absence of VPAC1 signaling. With the exception of the Rikenellaceae RC9 gut group, these taxa were primarily members of the Gram-positive 
families Lachnospiraceae and Ruminococcaceae. In contrast, those 26 OTUs detected exclusively in samples from VPAC1 KO mice were dominated by four main OTUs, two of which annotated to Clostridium sensu stricto (family Clostridiaceae), one to Clostridioides difficile M68 (family Peptostreptococcaceae), and one to [Ruminococcus] gnavus group (family Lachnospiraceae), all of which correspond to Gram-positive bacteria (Figure 3D). Keeping in mind that mice of different genotypes were co-housed littermates, with similar environmental exposures, these taxa were either completely eliminated or present below the limit of detection in WT or HET mice but allowed to colonize or proliferate in the absence of VPAC1 signaling.

A similar analysis within the VPAC2 colony revealed a single OTU present at a significantly different relative abundance dependent on genotypes (Supplementary Figure 1). Specifically, an OTU annotated to family Prevotellaceae NK3B31 group was found at greater relative abundance in VPAC2 KO mice compared to WT and HET littermates (FDR-adjusted $p=5.4 \times 10^{-5}$ ). Focused analysis of taxa detected only in samples from VPAC2 WT and HET, or VPAC2 KO, mice revealed a relatively conserved community structure between genotypes. Collectively, those OTUs found selectively in VPAC2 WT and HET or KO mice accounted for a remarkably small proportion ( $<0.01 \%$ on average) of each bacterial community.

To visualize $\beta$-diversity among the different groups, principal coordinate analysis (PCoA) was performed. When viewed together and based on either the Bray-Curtis or Jaccard similarities (SupplementaryFigure 2A and 2B), the two colonies of mice (VPAC1 and VPAC2) cluster separately along PCo2, while the VPAC1 $\mathrm{KO}$ samples cluster distinctly from the other VPAC1 genotypes along PCo1. As we are primarily interested in genotype-dependent differences within either colony, rather than differences between the VPAC1 and VPAC2 colonies, we repeated the PCoA with the two colonies independently, to remove inter-colony differences as a source of variability. As before, the VPAC1 colony separated into two very distinct clusters consisting of either KO or WT and HET samples, with the latter clustering much more tightly than the former (Figure 4A). These differences were confirmed statistically using permutational multivariate ANOVA (PERMANOVA). Based on Bray-Curtis similarities, there was an overall significant difference between genotypes, while pairwise comparisons indicated that the significant differences were between WT and KO ( $p=0.0001 ; \mathrm{F}=34.2)$, and between HET and KO ( $p=0.0001 ; \mathrm{F}=30.7)$, while there was no difference between VPAC1 WT and HET mice $(p=0.42 ; \mathrm{F}=0.9)$ (Figure 4B). Similarly, PERMANOVA based on Jaccard similarities found an overall significant difference, dominated by changes between WT or HET compared to KO mice.

In contrast, focused comparisons of the different genotypes within the VPAC2 colony revealed much more subtle differences in community structure. While the three genotypes overlapped substantially on PCoA using either Bray-Curtis (Figure 4C) or Jaccard (Figure 4D) similarities, PERMANOVA detected significant differences between WT and KO mice $(p=0.0001 ; \mathrm{F}=11.2)$ and between WT and HET mice ( $p$ $=0.005 ; F=6.1)$ using Bray-Curtis similarities. Of note, comparison of Jaccard similarities revealed the same differences, however the $\mathrm{F}$ values associated with those differences were much lower $(p=0.0001 ; \mathrm{F}$ $=2.8$ for WT vs. KO and ( $p=0.0004 ; \mathrm{F}=2.6$ for WT vs. HET), suggesting that the detected differences in community structure are primarily due to differences in the relative abundance of shared taxa rather than 
the selective presence or absence of taxa within a certain genotype. Collectively, we interpret these data as evidence that functional VPAC1 is essential for maintenance of the normal GM structure, whereas VPAC2 is much more dispensable with regard to GM community structure, richness, and diversity.

Lastly, we compared the genotype-dependent differences observed in the VPAC1 colony with the previously reported differences between mice deficient for VIP (the ligand for VPAC1 and VPAC2) and WT littermates (deposited in the NCBI Sequence Read Archive as BioProject \#PRJNA575206). If the differences in community structure in the absence of VPAC1 signaling are similar to those seen in the absence of functional VIP, a direct ligand:receptor effect becomes much more likely. Of the 94 OTUs identified as being present at statistically different relative abundance in VPAC1 KO mice, 11 were not detected at all in the VIP study, and 15 were detected in that study but were rare (present in $<10 \%$ of samples) or present at extremely low relative abundance (Figure 5A; Table 2, column $L$ ). Of the remaining OTUs, 59 of 68 (86.7\%) were consistent with significant differences observed in VIP-deficient mice, while there was either no difference observed in VIP-deficient mice in 8 of $68(11.8 \%)$ of the OTUs, and a contrasting difference in the VIP study in only one taxon (1.5\%), Roseburia sp. (Figure 5B). Taken as a whole, these data provide compelling evidence that VIP binding of VPAC1 is responsible for maintenance of the normal GM structure, and that in the absence of either of those factors, the GM undergoes characteristic changes via as yet undefined mechanisms.

\section{Discussion}

This report provides evidence that genetic deletion of mouse VIP receptors results in significant phylogenetic differences ( $\beta$-diversity) in fecal microbiota compared to wild type controls, irrespective of sex. VPAC1 KO fecal samples, which showed more substantial $\beta$-diversity changes compared to VPAC2 $\mathrm{KO}$ animals, also showed reductions in richness and a-diversity. Moreover, altered OTUs from VPAC1 fecal samples, including Bacteroides, Parabacteroides, Lactobacillus and Parasutterella, shared significant agreement to altered OTUs from VIP KO samples previously published ${ }^{19}$. In total, these data support that VIP receptor deficiency is associated with changes in gut microbiota ecology to different magnitudes and signaling mechanisms, highlighting their importance for gut health and homeostasis.

The influence of VIP within the GIT is dependent on the expression profile of its receptors, VPAC1 and VPAC2. In mice, Jayawardena et al. reported exclusive VPAC1 expression in the jejunum and ileum, with a 300-fold higher mRNA expression level for VPAC1 compared to VPAC2 or VIP's low-affinity receptor, pituitary adenylyl cyclase activating polypeptide 1 (PAC1) in the proximal and distal colon. Mucosal scrapings of intestinal epithelial cells reveal similar VPAC1 protein expression throughout the mouse intestines ${ }^{5}$. Earlier radioactive VIP labeled protein studies and VPAC2-specific binding studies support

VPAC1 as the predominant VIP receptor throughout the small and large intestines ${ }^{28,35}$. In humans, VPAC1 is also the predominant VIP receptor in sigmoid colon as assessed at the RNA and protein levels, with less but equivalent expression of all three VIP receptors in the small intestines ${ }^{5}$. Moreover, human HT-29 and Caco-2 intestinal epithelial cell lines express high levels of functional VPAC1 receptors ${ }^{36,37}$. 
Initial VPAC1 subcellular localization studies suggested a basolateral position for VPAC1 in rat, rabbit and human IECs ${ }^{38}$, while a more recent report showed murine VPAC1 expression co-localizing with the apical (e.g. villin), but not basolateral (e.g. $\mathrm{Na}^{+} / \mathrm{K}^{+}$ATPase), IEC marker ${ }^{30}$. These different findings could be explained by the heterogenous makeup of IECs consisting of absorptive (e.g. enterocytes and $\mathrm{M}$ cells) versus secretory (e.g. Goblet and Tuft) cell types, which are constantly renewed every $\approx 7$ days from differentiating crypt progenitor cells that migrate to the villus ${ }^{39}$. Interestingly, VPAC1 can be targeted to the nuclear membrane, which may further alter signaling effects dependent on its subcellular localization (e.g. apical, basolateral and/or nuclear) ${ }^{40}$. The present study supports VPAC1 as the predominant intestinal VIP receptor as its genetic deletion manifested in a more substantial change to the fecal microbiota composition compared to WT littermate controls or the VPAC2 colony. Furthermore, altered fecal bacterial taxa from VPAC1 KO mice presented significant similarities to those previously published by our group from VIP KO animals ${ }^{19}$, suggesting a bona fide VIP $\rightarrow$ VPAC1 signaling pathway in IECs regulating the stability and diversity of the gut microbiota.

Intestinal IECs provide physical and chemical barriers that protect the host from the gut microbiota, while absorbing essential nutrients for host survival ${ }^{41}$. Breakdown of the intestinal epithelial barrier and gut microbiota dysbiosis are linked to serious health consequences, including inflammatory bowel disease ${ }^{42}$, type II diabetes ${ }^{43}$ and obesity ${ }^{44}$. Evidence linking VIP $\rightarrow$ VPAC1 signaling to IEC homeostasis and intestinal barrier integrity has been provided by multiple studies. First, observations in the 1980's demonstrated VIPergic neurons in the intestinal mucosa, showing VIP accumulation in the lumen when stimulated by cholera toxin ${ }^{45,46}$. More recent studies have confirmed that VIPergic nerve fibers innervate all layers of the intestinal tissue and account for nearly $50 \%$ of enteric neurons ${ }^{12}$. Moreover, a 2020 report delineated VIPergic nerves within the intestinal mucosa co-localizing with the $\beta$-III-tubulin neuronal marker ${ }^{30}$. Second, VIP KO mice possess fewer proliferating, mucin-expressing Goblet cells, compared to WT controls. Morphologically, VIP deficient mice manifested shorter and wider intestinal crypts with impaired barrier function as FITC-dextran enemas demonstrated greater fluorescence in the vasculature compared to control mice. Exogenously added VIP to VIP KO mice partially rescued the above phenotypes, and the authors concluded that the Goblet-specific defect and intesinal barrier ultrastructural changes were unlikely explained by developmental abnormalities ${ }^{14}$. Third, Waschek's group published findings that VIP-deficient mice had morphological defects within the GIT, including shorter, but heavier intestines resembling a "rubbery" appearance, due to a thickening of the muscularis propria. This group also reported a reduced mucus secretion potential from Goblet cells in VIP KO mice, consistent with the above study ${ }^{12}$. Fourth, older investigations contribute supportive evidence showing that VIP signaling increases proliferation of IECs from in vivo and in vitro experiments ${ }^{47,48}$. Lastly, a report using an organotypic mouse intestinal slice model that maintained three-dimensional intestinal architecture provided evidence that blocking VIP signaling with a pan-VPAC antagonist substantially reduced the number of mucus-producing Goblet cells in intestinal crypts, suggesting that VIP signaling is important for Goblet cell differentiation and survival ${ }^{49}$. Couple the above intesinal IEC changes with other known intestinal VIP effects including: 1.) regulation of luminal water and electrolyte levels ${ }^{50}$, 2.) peristalsis rate 
51 , 3.) metabolic gut hormone secretion, ${ }^{1}$, 4.) anti-inflammatory effects $14,52,53$ and 5.) antimicrobial activity ${ }^{54}$, it becomes apparent that there is potential for an amalgamation of environmental changes within the intestines from dysregulated VIP signaling. Genetic ablation of either VIP ${ }^{19}$ or VPAC1 (present study) results in significant and similar gut microbiota compositional changes and reduction in microbiota diversity. In aggregate, we propose a neuronal-IEC VIP $\rightarrow$ VPAC1 signaling circuitry is critical to maintaining intestinal health, goblet cell homeostasis and a stable gut microbiota.

$\mathrm{VIP} \rightarrow \mathrm{VPAC} 1$ signaling in IECs maintains the expression of several genes. One of these gene targets is the intestine-specific protein, called caudal-related homeobox transcription factor 2 (CDX2), which is significantly reduced in VIP KO intestinal tissue ${ }^{14}$. CDX2 is critical for epithelial progenitor self-renewal and differentiation, and CDX2 conditional knockouts transition to a gastric-like appearance ${ }^{55}$. Wu X. et al. showed that VIP KO mice had fewer Goblet cells and abnormally elevated proliferative capacity in cells the closer they were to intestinal crypts, providing evidence that a VIPàCDX2 axis may influence the proliferation/differentiation balance of epithelial progenitors ${ }^{14}$. CDX2 transcriptionally activates MUC2 and Tff3, which are major gene products in mucus ${ }^{56,57}$. VIP deficient mice show reduced MUC2 expression/secretion in intestine, and MUC2 deficiency leads to a depletion of the butyrate-producing Ruminococcaceae Family as observed in the present study from VPAC1 KO mice, and in VIP KO animals previously published ${ }^{19}$. CDX2 also regulates tight junction genes that contribute to intestinal barrier function. During intestinal inflammatory insult either by hypoxia/LPS, TNBS or Citrobacter rodentium infections, endogenously added VIP or a more stable recombinant VIP analogue (rVIPa), results in restored levels and subcellular targeting of tight junction proteins, Claudin-3, occludin and ZO-1 by inhibition of PKC€ and myosin light chain kinase (MLCK) ${ }^{58-60}$. These data support a $\mathrm{VIP} \rightarrow \mathrm{VPAC} 1 \rightarrow \mathrm{CDX} 2 \rightarrow \mathrm{MUC} 2$ pathway in intestinal progenitor cells and Goblet cells that maintains epithelial barrier integrity, mucus secretion, tight junctions and gut microbiota stability. Future research employing VIP receptor conditional knockouts and fate mapping will be instrumental in better understanding this important molecular pathway.

To date, there exists significant disagreement as to whether VIP deficiency results in greater or less susceptibility to intestinal inflammation, including models of IBD ${ }^{61,62}$. Our group and others have suggested that genetic deletion of VIP influencing gut microbiota changes, along with other environmental factors such as diet and housing conditions, may tip the scales towards an anti- versus pro-inflammatory intestinal tone ${ }^{14,19}$. An additional possibility could be a delicate balance between mutually opposing VIP $\rightarrow$ VPAC1 $\rightarrow \mathrm{CDX} 2$ and NF-KB actions regulating proinflammatory genes. Proinflammatory cytokines (e.g. TNF-a) can increase p50/p65 NF-KB heterodimers that compete away activating p50/p50 NF-kB homodimers at the CDX2 promoter thereby inhibiting its expression. In opposition to this, CDX2 can limit p50/p65 DNA binding and upregulation of proinflammatory genes, while bolstering its own expression ${ }^{63}$. VIP $\rightarrow$ VPAC1 signaling inhibits $p 50 / p 65$ NF-kB nuclear localization by stabilizing IKB in inflamed intestinal tissue that blocks LPS-induced TNF-a that in turn would maintain CDX2 expression ${ }^{59,64}$. Consistent with this mechanism, human IBD tissue samples show reduced 
VIPergic nerve density and CDX2 expression, with elevated NF-kB p50/p65 activation ${ }^{65,66}$. Furthermore, CDX2 heterozygous mice are more susceptible to DSS induced colitis compared to controls ${ }^{67}$. CDX2's transcriptional activity regulating genes that control barrier integrity, cellular differentiation and apoptosis are regulated in turn by MAP kinases. Phosphorylation of CDX2 on serine 60 by ERK1/2 decreases its transcriptional activity, while phosphorylation by p38 increases its transcriptional activity. VIP $\rightarrow$ VPAC1 signaling could switch CDX2 from transcriptionally active to transcriptionally repressive through its ability to activate both ERK1/2 and p38 pathways. Disentangling the VIP $\rightarrow \mathrm{VPAC} 1 \rightarrow \mathrm{CDX} 2$ and NF-KB signaling circuitry in IECs will be critical in better understanding the intricate balance between intestinal barrier integrity, inflammation and gut bacterial ecology.

Both VPAC1 and VPAC2 are expressed by various immune cells within the intestines, including macrophages and T cells ${ }^{68}$. In T cells, VPAC1 has been reported by our group and others to be expressed at higher levels than VPAC2 in naïve T lymphocytes, but downregulated during T cell activation ${ }^{69}$. Vomhoff-DeKrey et al. went further to describe the plasticity of VPAC1 expression returning to naïve levels in antigen-specific CD8 T cell (OT-I transgenic) primary memory cells, that failed to return to naïve levels after a secondary activation using a $\mathrm{T}_{\mathrm{h} 1}$ pathogen in vivo infection model ${ }^{3}$. VIP $\rightarrow$ VPAC1 signaling can induce tolerogenic dendritic cells, which present commensal peptides to naïve $T$ cells controlling $T$ cell effector/regulator ratios, important in mucosal immunity ${ }^{52}$. It has been shown that differences in commensal peptide antigen presentation to T cells within the "mucosal firewall" can have profound influences on gut microbiota composition ${ }^{20,70}$. On the other hand, VPAC2 expression is upregulated, while VPAC1 is downregulated on $\mathrm{Th}_{2}$ effector cells, thereby altering how VIP signals are interpreted by $\mathrm{T}$ lymphocytes based on their activation status ${ }^{71}$. Another immune cell population that expresses VPAC2 and plays a pivotal role in maintaining epithelial barrier integrity is type-3 innate lymphoid cells (ILC3). Microbiota-associated molecular patterns activate dendritic cells and macrophages to produce IL-23, which binds to receptors on CCR6 ${ }^{+}$ILC3 to secrete IL-22. IEC respond to ILC3-derived IL-22 by secreting the anti-microbial peptide, Reg3g into the lumen, while blocking lipid transporter gene, Fabp2. In 2020, Talbot et al. eloquently demonstrated that food intake and/or Citrobacter rodentium oral gavage resulted in barrier integrity breakdown with greater bacterial translocation to the spleen and liver in a VIPdependent manner ${ }^{30}$. This study showed that VPAC2 crosslinking by VIP on ILC3 caused IL-22 inhibition that simultaneously reduced $R e g 3 g$ downregulation while increasing the expression of Fabp2. Consistent with the present study, VPAC2 conditional KO mice in ILC3 showed fluctuations in fecal and cecum microbiota composition ${ }^{30}$.

VIP shares $68 \%$ amino acid identity with pituitary adenylate cyclase activating polypeptide (PACAP), which also binds to both VPAC1 and VPAC2 receptors with similar affinity to VIP ( $\approx 1 \mathrm{nM} \mathrm{Kd})$. Both neuropeptides are delivered to the GIT by the peripheral nervous system and PACAP deficient mice also revealed similar fecal microbiota changes with respect to enrichments in Gram negative bacteria, including genera from Bacteroidetes and Proteobacteria ${ }^{72}$. In contrast, VIP and VPAC1 deficient mice showed similar reductions in the Gram positive Lachnospiraceae and Ruminococcaceae families that 
were not observed from PACAP knockout mice. Based on the high extent of agreement between VIP- and VPAC1-deficient fecal microbiota compositional changes, and a lack of effect on the Firmicutes phylum reported for PACAP deficient mice, we conclude that there is little to no compensation from PACAP on microbiota composition in either VIP- or VPAC1-deficient mice. We would further posit that these reports bolster the notion of a VIP $\rightarrow$ VPAC1 signaling axis within the gut that plays a pivotal role in maintaining the stability and diversity of the gut microbiota.

It is difficult to extrapolate the consequence to the host due to the gut microbiota changes observed in VIP receptor deficient mice. However, this report supports a consistency in gut microbiota structural changes between VPAC1 deficient mice (current report) and VIP deficient mice ${ }^{19}$. Consistent blooms and depletions at the OTU level were observed that favor LPS positive, Gram-negative (e.g. Bacteroides, Parabacteroides, Parasutterella and Escherichia) bacteria that would be expected to result in inflammation. VIP signaling through VPAC1 has historically been viewed to drive most of the antiinflammatory effects observed by VIP ${ }^{73}$, and with the recent finding that VIP signaling blocks LPSinduced inflammasome formation, we propose that the loss of VIP $\rightarrow$ VPAC1 signaling provides an environment for elevated LPS from Gram-negative blooms leading to local and possibly systemic inflammation. Research is now ongoing in our research group to assess this possibility.

We have provided 16S rRNA sequencing data from both VPAC1 and VPAC2 KO fecal samples demonstrating statistically significant gut bacterial compositional changes. VPAC1 signaling, presumably in IECs, resulted in more severe alterations in gut microbiota structure and diversity loss compared to VPAC2 deficient mice. Moreover, more than $86 \%$ of the gut microbiota genera changes were consistent between VPAC1 deficient samples (present study) and VIP deficient samples (previously published) implicating the VIP $\rightarrow$ VPAC1 signaling pathway as a major driving force behind gut microbiota stability. Our results place the VIP receptors as pivotal signaling GPCRs regulating intestinal homeostasis and gut microbiota stability. Future research will undoubtably shed greater light on this important signaling axis within the GIT.

\section{Methods}

\section{Mice}

VPAC1 and VPAC2 HET breeders were kind gifts from Professors Sue O'Dorisio ${ }^{74}$ and Anthony Harmar 31 , and both mouse lines are commercially available (Jackson Laboratory; Bar Harbor, ME). Mice were bred at North Dakota State University, and research was approved by the NDSU Institutional Animal Care and Use Committee (IACUC). All studies within this report were conducted in accordance of all state and federal regulations and guidelines. Mice were housed in polycarbonate cages containing Alpha-Dri paper bedding (Animal Care Systems, Centennial, CO), with access to 5001 LabDiet mouse chow (St. Louis, MO) and tap water ad libitum with a $12 \mathrm{hr}$ light/dark cycle. Mice were ear clipped for identification and tail biopsies collected for PCR genotyping ${ }^{31,74}$. 


\section{Mouse genotyping}

DNA was extracted from tail biopsies using Sigma-Aldrich extraction kit (St. Louis, MO) as previously published ${ }^{19}$. Briefly, PCR reactions were performed with a unique forward wild type primer and a common reverse primer (WT reactions), or a unique forward mutant primer and a common reverse primer (KO reactions). PCR reactions (20 $\mu \mathrm{l}$ ) containing 1x GoTaq ${ }^{\circledR}$ G2 master mix (Promega, Madison, WI), primers $(312.5 \mathrm{nM})$ and $2 \mu$ of DNA template $(1 / 20$ dilution in TE) or nuclease-free water were amplified by PCR with the following parameters: $94{ }^{\circ} \mathrm{C}(3: 00)+\left[94^{\circ} \mathrm{C}(0: 15), 62^{\circ} \mathrm{C}(0: 45), 72^{\circ} \mathrm{C}(0: 45)\right] \times 40$. Reactions were separated by agarose gel electrophoresis, visualized by UV light $(254 \mathrm{~nm})$ using EZ-Vision dye (Amresco, Radnor, PA) and photographed with a digital camera (Alpha Innotech).

\section{DNA extraction}

Fecal DNA was extracted using PowerFecal kits (Qiagen) according to the manufacturer's instructions, with the exception that, rather than performing the initial homogenization of samples using the vortex adapter described in the protocol, samples were homogenized in the provided bead tubes using a TissueLyser II (Qiagen) for three minutes at 30/sec, before proceeding according to the protocol and eluting in $100 \mu \mathrm{L}$ of elution buffer (Qiagen). DNA yields were quantified via fluorometry (Qubit 2.0, Invitrogen, Carlsbad, CA) using quant-iT BR dsDNA reagent kits (Invitrogen).

\section{S rRNA library preparation and sequencing}

Extracted fecal DNA was processed at the University of Missouri DNA Core Facility. Bacterial 16S rRNA amplicons were constructed via amplification of the V4 region of the 16S rRNA gene with universal primers (U515F/806R) previously developed against the V4 region, flanked by Illumina standard adapter sequences ${ }^{75,76}$. Oligonucleotide sequences are available at proBase ${ }^{77}$. Dual-indexed forward and reverse primers were used in all reactions. PCR was performed in $50 \mu \mathrm{L}$ reactions containing $100 \mathrm{ng}$ metagenomic DNA, primers ( $0.2 \mu \mathrm{M}$ each), dNTPs (200 $\mu \mathrm{M}$ each), and Phusion high-fidelity DNA polymerase (1U). Amplification parameters were $98^{\circ} \mathrm{C}^{(3: 00)}+\left[98^{\circ} \mathrm{C}^{(0: 15)}+50^{\circ} \mathrm{C}^{(0: 30)}+72^{\circ} \mathrm{C}^{(0: 30)}\right] \times 25$ cycles $+72^{\circ} \mathrm{C}^{(7: 00)}$. Amplicon pools ( $5 \mu \mathrm{L} /$ reaction) were combined, thoroughly mixed, and then purified by addition of Axygen Axyprep MagPCR clean-up beads to an equal volume of $50 \mu \mathrm{L}$ of amplicons and incubated for 15 minutes at room temperature. Products were then washed multiple times with $80 \%$ ethanol and the dried pellet resuspended in $32.5 \mu \mathrm{L}$ EB buffer, incubated for two minutes at room temperature, and then placed on the magnetic stand for five minutes. The final amplicon pool was evaluated using the Advanced Analytical Fragment Analyzer automated electrophoresis system, quantified using quant-iT HS dsDNA reagent kits, and diluted according to Illumina's standard protocol for sequencing on the MiSeq instrument.

\section{Informatics analysis}

Read merging, clustering, and annotation of DNA sequences was performed at the University of Missouri Informatics Research Core Facility. Paired DNA sequences were merged using FLASH software ${ }^{78}$, and 
removed if found to be far from the expected length of 292 bases after trimming for base quality of 31 . Cutadapt ${ }^{79}$ (https://github.com/marcelm/cutadapt) was used to remove the primers at both ends of the contig and cull contigs that did not contain both primers. The usearch ${ }^{80}$ fastq_filter command (http://drive5.com/usearch/manual/cmd_fastq_filter.html) was used for quality trimming of contigs, rejecting those for which the expected number of errors is greater than 0.5 . All contigs were trimmed to 248 bases and shorter contigs are removed. The Qiime ${ }^{81} 1.9$ command split_libraries_fastq.py was used to demultiplex the samples. The outputs for all samples were combined into a single file for clustering. The uparse ${ }^{82}$ method (http://www.drive5.com/uparse/) was used to both cluster contigs with $97 \%$ identity and remove chimeras. Taxonomy was assigned to selected OTUs using BLAST ${ }^{83}$ against the SILVA database $v 132^{84}$ of 16 S rRNA sequences and taxonomy.

\section{Statistics}

Statistical comparisons were performed within each colony (i.e., VPAC1 and VPAC2) separately. Comparison of univariate metrics (e.g., richness, a-diversity) commenced with testing for normality and equal variance using the Shapiro-Wilk and Brown-Forsyth methods, respectively. For normally distributed data, one- or two-factor ANOVA was performed to test for differences between genotypes, or interactions between genotype and sex. Kruskall-Wallis ANOVA on ranks was used for non-normally distributed data. These tests were performed using SigmaPlot 14.0 (Systat software, Inc.). For serial testing of genotypedependent differences in the relative abundance of OTUs within each colony, and determination of FDRadjusted $p$ values, MetaboAnalyst software ${ }^{85}$ was used. Raw $p$ values were also compared to critical $p$ values determined using the method of Benjamini and Hochberg, to ensure all differences withstood correction for multiple tests. One-way permutational multivariate analysis of variance (PERMANOVA) was used to perform principal coordinate analysis (PCoA) and test for differences in $\beta$-diversity within each colony. Both PCoA and PERMANOVA were performed using relatively weighted (Bray-Curtis) and unweighted (Jaccard) similarities as a form of validation, but also to determine the nature of any detected differences. All multivariate analysis were performed using Past software ${ }^{86}$.

\section{Declarations}

\section{Data Availability}

This data set has been registered under BioProject ID PRJNA679452 and can be accessed by the following URL http://www.ncbi.nlm.nih.gov/bioproject/679452.

\section{Author Contributions}

M.B. managed the daily operations of the mouse vivarium that included daily documentation, topping off food and water, weekly cage changes, weaning, tail biopsy collection, DNA extraction and PCR genotyping and fecal collections. M.B. assisted with the writing of the manuscript as part of his doctoral dissertation. J.W. provided expert experimental design review and interpretation of results. A.E. provided 
the DNA isolation from fecal samples, $16 \mathrm{~S}$ rRNA sequencing and authored the results sections, including the tables and figure representations. G.D. designed, interpreted, and wrote the manuscript. All authors edited the final manuscript version.

\section{Competing Interest Statement}

Authors do not have competing interests with respect to the content of this study.

\section{References}

1. Martin, B. et al. Vasoactive intestinal peptide-null mice demonstrate enhanced sweet taste preference, dysglycemia, and reduced taste bud leptin receptor expression. Diabetes.59, 1143-1152 https://doi.org/10.2337/db09-0807 (2010).

2. De Vadder, F., Plessier, F., Gautier-Stein, A. \& Mithieux, G. Vasoactive intestinal peptide is a local mediator in a gut-brain neural axis activating intestinal gluconeogenesis. Neurogastroenterol Motil.27, 443-448 https://doi.org/10.1111/nmo.12508 (2015).

3. Vomhof-DeKrey, E. E., Haring, J. S. \& Dorsam, G. P. Vasoactive intestinal peptide receptor 1 is downregulated during expansion of antigen-specific CD8 T cells following primary and secondary Listeria monocytogenes infections. J Neuroimmunol.234, 40-48 https://doi.org/10.1016/j.jneuroim.2011.02.002 (2011).

4. Dorsam, G. P., Benton, K., Failing, J. \& Batra, S. Vasoactive intestinal peptide signaling axis in human leukemia. World J Biol Chem.2, 146-160 https://doi.org/10.4331/wjbc.v2.i6.146 (2011).

5. Jayawardena, D. et al. Expression and localization of VPAC1, the major receptor of vasoactive intestinal peptide along the length of the intestine. American journal of physiology. Gastrointestinal and liver physiology.313, G16-g25 https://doi.org/10.1152/ajpgi.00081.2017 (2017).

6. Said, S. I. \& Mutt, V. Polypeptide with broad biological activity: isolation from small intestine. Science.169, 1217-1218 (1970).

7. Nyberg, B., Angelin, B. \& Einarsson, K. Somatostatin does not block the effect of vasoactive intestinal peptide on bile secretion in man. Eur J Clin Invest.22, 60-66 https://doi.org/10.1111/j.13652362.1992.tb01937.x (1992).

8. Barbezat, G. O. The effect of luminal and hormonal factors on small intestinal water and electrolyte transport. Schweiz Med Wochenschr.116, 946-949 (1986).

9. Barbezat, G. O. \& Grossman, M. I. Intestinal secretion: stimulation by peptides. Science.174, 422-424 https://doi.org/10.1126/science.174.4007.422 (1971).

10. Benarroch, E. E. Enteric nervous system: functional organization and neurologic implications. Neurology.69, 1953-1957 https://doi.org/10.1212/01.wnl.0000281999.56102.b5 (2007).

11. Van Geldre, L. A. \& Lefebvre, R. A. Interaction of NO and VIP in gastrointestinal smooth muscle relaxation. Curr Pharm Des.10, 2483-2497 https://doi.org/10.2174/1381612043383890 (2004). 
12. Lelievre, V. et al. Gastrointestinal dysfunction in mice with a targeted mutation in the gene encoding vasoactive intestinal polypeptide: a model for the study of intestinal ileus and Hirschsprung's disease. Peptides28, 1688-1699, doi:S0196-9781(07)00171-4 [pii]10.1016/j.peptides. 2007.05.006 (2007).

13. Nussbaum, J. C. et al. Type 2 innate lymphoid cells control eosinophil homeostasis. Nature.502, 245-248 https://doi.org/10.1038/nature12526 (2013).

14. Wu, X. et al. Vasoactive intestinal polypeptide promotes intestinal barrier homeostasis and protection against colitis in mice. PloS one.10, e0125225 https://doi.org/10.1371/journal.pone.0125225 (2015).

15. Turnbaugh, P. J. et al. The effect of diet on the human gut microbiome: a metagenomic analysis in humanized gnotobiotic mice. Sci Trans/ Med.1, 614 https://doi.org/10.1126/scitransImed.3000322 (2009).

16. Hooper, L. V. \& Gordon, J. I. Commensal host-bacterial relationships in the gut. Science.292, 11151118 (2001).

17. Vaishampayan, P. A. et al. Comparative metagenomics and population dynamics of the gut microbiota in mother and infant. Genome Biol Evol.2, 53-66 https://doi.org/10.1093/gbe/evp057 (2010).

18. Le Doare, K., Holder, B., Bassett, A. \& Pannaraj, P. S. Mother's Milk: A Purposeful Contribution to the Development of the Infant Microbiota and Immunity. Front Immunol.9, 361 https://doi.org/10.3389/fimmu.2018.00361 (2018).

19. Bains, M. et al. Vasoactive Intestinal Peptide Deficiency Is Associated With Altered Gut Microbiota Communities in Male and Female C57BL/6 Mice. Frontiers in microbiology.10, 2689 https://doi.org/10.3389/fmicb.2019.02689 (2019).

20. Khan, A. A. et al. Polymorphic Immune Mechanisms Regulate Commensal Repertoire. Cell Rep.29, 541-550544 https://doi.org/10.1016/j.celrep.2019.09.010 (2019).

21. Turnbaugh, P. J., Backhed, F., Fulton, L. \& Gordon, J. I. Diet-induced obesity is linked to marked but reversible alterations in the mouse distal gut microbiome. Cell host \& microbe.3, 213-223 https://doi.org/10.1016/j.chom.2008.02.015 (2008).

22. Carmody, R. N. et al. Diet dominates host genotype in shaping the murine gut microbiota. Cell host \& microbe.17, 72-84 https://doi.org/10.1016/j.chom.2014.11.010 (2015).

23. Rothschild, D. et al. Environment dominates over host genetics in shaping human gut microbiota. Nature.555, 210-215 https://doi.org/10.1038/nature25973 (2018).

24. Rawls, J. F., Mahowald, M. A., Ley, R. E. \& Gordon, J. I. Reciprocal gut microbiota transplants from zebrafish and mice to germ-free recipients reveal host habitat selection. Cell.127, 423-433 https://doi.org/10.1016/j.cell.2006.08.043 (2006).

25. Goodrich, J. K. et al. Genetic Determinants of the Gut Microbiome in UK Twins. Cell host \& microbe.19, 731-743 https://doi.org/10.1016/j.chom.2016.04.017 (2016). 
26. Gum, J. R. Jr., Hicks, J. W., Toribara, N. W., Siddiki, B. \& Kim, Y. S. Molecular cloning of human intestinal mucin (MUC2) cDNA. Identification of the amino terminus and overall sequence similarity to prepro-von Willebrand factor. J Biol Chem.269, 2440-2446 (1994).

27. Wu, M. et al. The Dynamic Changes of Gut Microbiota in Muc2 Deficient Mice. International journal of molecular sciences.19, https://doi.org/10.3390/ijms19092809 (2018).

28. Harmar, A. J. et al. Distribution of the VPAC2 receptor in peripheral tissues of the mouse. Endocrinology.145, 1203-1210 https://doi.org/10.1210/en.2003-1058 (2004).

29. Roager, H. M. et al. Colonic transit time is related to bacterial metabolism and mucosal turnover in the gut. Nat Microbiol.1, 16093 https://doi.org/10.1038/nmicrobiol.2016.93 (2016).

30. Talbot, J. et al. Feeding-dependent VIP neuron-ILC3 circuit regulates the intestinal barrier. Nature.579, 575-580 https://doi.org/10.1038/s41586-020-2039-9 (2020).

31. Harmar, A. J. et al. The VPAC(2) receptor is essential for circadian function in the mouse suprachiasmatic nuclei. Cell.109, 497-508 https://doi.org/10.1016/s0092-8674(02)00736-5 (2002).

32. Thaiss, C. A. et al. Transkingdom control of microbiota diurnal oscillations promotes metabolic homeostasis. Cell.159, 514-529 https://doi.org/10.1016/j.cell.2014.09.048 (2014).

33. Hieke, A. C., Hubert, S. M. \& Athrey, G. Circadian disruption and divergent microbiota acquisition under extended photoperiod regimens in chicken. PeerJ.7, e6592 https://doi.org/10.7717/peerj.6592 (2019).

34. Weger, B. D. et al. The Mouse Microbiome Is Required for Sex-Specific Diurnal Rhythms of Gene Expression and Metabolism. Cell metabolism.29, 362-382368 https://doi.org/10.1016/j.cmet.2018.09.023 (2019).

35. Nau, R., Ballmann, M. \& Conlon, J. M. Binding of vasoactive intestinal polypeptide to dispersed enterocytes results in rapid removal of the NH2-terminal histidyl residue. Mol Cell Endocrinol.52, 97103 https://doi.org/10.1016/0303-7207(87)90102-x (1987).

36. Summers, M. A., O'Dorisio, M. S., Cox, M. O., Lara-Marquez, M. \& Goetzl, E. J. A lymphocyte-generated fragment of vasoactive intestinal peptide with VPAC1 agonist activity and VPAC2 antagonist effects. J Pharmacol Exp Ther.306, 638-645 https://doi.org/10.1124/jpet.103.050583jpet.103.050583[pii] (2003).

37. Anderson, C. M., Mendoza, M. E., Kennedy, D. J., Raldua, D. \& Thwaites, D. T. Inhibition of intestinal dipeptide transport by the neuropeptide VIP is an anti-absorptive effect via the VPAC1 receptor in a human enterocyte-like cell line (Caco-2). Br J Pharmacol.138, 564-573 https://doi.org/10.1038/sj.bjp.0705049 (2003).

38. Dharmsathaphorn, K., Mandel, K. G., Masui, H. \& McRoberts, J. A. Vasoactive intestinal polypeptideinduced chloride secretion by a colonic epithelial cell line. Direct participation of a basolaterally localized $\mathrm{Na}+, \mathrm{K}+, \mathrm{Cl}$ - cotransport system. J Clin Invest.75, 462-471 https://doi.org/10.1172/jci111721 (1985).

39. O'Leary, C. E., Schneider, C. \& Locksley, R. M. Tuft Cells-Systemically Dispersed Sensory Epithelia Integrating Immune and Neural Circuitry. Annu Rev Immunol.37, 47-72 
https://doi.org/10.1146/annurev-immunol-042718-041505 (2019).

40. Yu, R. et al. The palmitoylation of the N-terminal extracellular Cys37 mediates the nuclear translocation of VPAC1 contributing to its anti-apoptotic activity. Oncotarget.8, 42728-42741 https://doi.org/10.18632/oncotarget.17449 (2017).

41. Pelaseyed, T. et al. The mucus and mucins of the goblet cells and enterocytes provide the first defense line of the gastrointestinal tract and interact with the immune system. Immunol Rev.260, 820 https://doi.org/10.1111/imr.12182 (2014).

42. Parikh, K. et al. Colonic epithelial cell diversity in health and inflammatory bowel disease. Nature.567, 49-55 https:// doi.org/10.1038/s41586-019-0992-y (2019).

43. Sharma, S. \& Tripathi, P. Gut microbiome and type 2 diabetes: where we are and where to go? J Nutr Biochem.63, 101-108 https://doi.org/10.1016/j.jnutbio.2018.10.003 (2019).

44. Chelakkot, C., Ghim, J. \& Ryu, S. H. Mechanisms regulating intestinal barrier integrity and its pathological implications. Exp Mol Med.50, 103 https://doi.org/10.1038/s12276-018-0126-x (2018).

45. Bitar, K. N., Said, S. I., Weir, G. C., Saffouri, B. \& Makhlouf, G. M. Neural release of vasoactive intestinal peptide from the gut. Gastroenterology.79, 1288-1294 (1980).

46. Cassuto, J., Fahrenkrug, J., Jodal, M., Tuttle, R. \& Lundgren, O. Release of vasoactive intestinal polypeptide from the cat small intestine exposed to cholera toxin. Gut.22, 958-963 https://doi.org/10.1136/gut.22.11.958 (1981).

47. Gamet, L. et al. Vasoactive intestinal peptide and forskolin regulate proliferation of the HT29 human colon adenocarcinoma cell line. Journal of cellular physiology.150, 501-509 https://doi.org/10.1002/jcp.1041500310 (1992).

48. See, N. A., Epstein, M. L., Dahl, J. L. \& Bass, P. The myenteric plexus regulates cell growth in rat jejunum. J Auton Nerv Syst.31, 219-229 https://doi.org/10.1016/0165-1838(90)90188-o (1990).

49. Schwerdtfeger, L. A. \& Tobet, S. A. Vasoactive intestinal peptide regulates ileal goblet cell production in mice. Physiol Rep.8, e14363 https://doi.org/10.14814/phy2.14363 (2020).

50. Hejna, M. et al. Serum levels of vasoactive intestinal peptide (VIP) in patients with adenocarcinomas of the gastrointestinal tract. Anticancer Res.21, 1183-1187 (2001).

51. Tomita, R. et al. Physiological studies on vasoactive intestinal peptide in rat small bowel isografts. Transplant Proc.36, 367-369 https://doi.org/10.1016/j.transproceed.2003.12.011 (2004).

52. Toscano, M. G. et al. Dendritic cells transduced with lentiviral vectors expressing VIP differentiate into VIP-secreting tolerogenic-like DCs. Molecular therapy: the journal of the American Society of Gene Therapy.18, 1035-1045 https://doi.org/10.1038/mt.2009.293 (2010).

53. Kimura, M., Masuda, T., Hiwatashi, N., Toyota, T. \& Nagura, H. Changes in neuropeptide-containing nerves in human colonic mucosa with inflammatory bowel disease. Pathology international.44, 624634 (1994).

54. El Karim, I. A., Linden, G. J., Orr, D. F. \& Lundy, F. T. Antimicrobial activity of neuropeptides against a range of micro-organisms from skin, oral, respiratory and gastrointestinal tract sites. $J$ 
Neuroimmunol.200, 11-16 https://doi.org/10.1016/j.jneuroim.2008.05.014 (2008).

55. Grainger, S., Savory, J. G. \& Lohnes, D. Cdx2 regulates patterning of the intestinal epithelium. Dev Biol.339, 155-165 https://doi.org/10.1016/j.ydbio.2009.12.025 (2010).

56. Shimada, T., Koike, T., Yamagata, M., Yoneda, M. \& Hiraishi, H. Regulation of TFF3 expression by homeodomain protein CDX2. Regul Pept.140, 81-87 https://doi.org/10.1016/j.regpep.2006.11.014 (2007).

57. Mesquita, P. et al. Human MUC2 mucin gene is transcriptionally regulated by $\mathrm{Cdx}$ homeodomain proteins in gastrointestinal carcinoma cell lines. J Biol Chem.278, 51549-51556 https://doi.org/10.1074/jbc.M309019200 (2003).

58. Morampudi, V. et al. Vasoactive intestinal peptide prevents PKC $\varepsilon$-induced intestinal epithelial barrier disruption during EPEC infection. American journal of physiology. Gastrointestinal and liver physiology.308, G389-402 https://doi.org/10.1152/ajpgi.00195.2014 (2015).

59. Xu, C. L., Guo, Y., Qiao, L., Ma, L. \& Cheng, Y. Y. Recombinant expressed vasoactive intestinal peptide analogue ameliorates TNBS-induced colitis in rats. World journal of gastroenterology.24, 706-715 https://doi.org/10.3748/wjg.v24.i6.706 (2018).

60. Conlin, V. S. et al. Vasoactive intestinal peptide ameliorates intestinal barrier disruption associated with Citrobacter rodentium-induced colitis. American journal of physiology. Gastrointestinal and liver physiology.297, G735-750 https://doi.org/10.1152/ajpgi.90551.2008 (2009).

61. Duffy, L. C. et al. Vasoactive intestinal peptide as a laboratory supplement to clinical activity index in inflammatory bowel disease. Dig Dis Sci.34, 1528-1535 (1989).

62. El-Salhy, M., Solomon, T., Hausken, T., Gilja, O. H. \& Hatlebakk, J. G. Gastrointestinal neuroendocrine peptides/amines in inflammatory bowel disease. World journal of gastroenterology.23, 5068-5085 https://doi.org/10.3748/wjg.v23.i28.5068 (2017).

63. Coskun, M., Troelsen, J. T. \& Nielsen, O. H. The role of CDX2 in intestinal homeostasis and inflammation. Biochim Biophys Acta.1812, 283-289 (2011).

64. Delgado, M. et al. Vasoactive intestinal peptide and pituitary adenylate cyclase-activating polypeptide inhibit tumor necrosis factor alpha transcriptional activation by regulating nuclear factor-kB and cAMP response element-binding protein/c-Jun. J Biol Chem.273, 31427-31436 (1998).

65. Rogler, G. et al. Nuclear factor kappaB is activated in macrophages and epithelial cells of inflamed intestinal mucosa. Gastroenterology.115, 357-369 https://doi.org/10.1016/s0016-5085(98)70202-1 (1998).

66. Sipos, F. et al. Regeneration associated growth factor receptor and epithelial marker expression in lymphoid aggregates of ulcerative colitis. Scand J Gastroenterol.45, 440-448 https://doi.org/10.3109/00365521003624144 (2010).

67. Calon, A. et al. Different effects of the $\mathrm{Cdx} 1$ and $\mathrm{Cdx} 2$ homeobox genes in a murine model of intestinal inflammation. Gut.56, 1688-1695 https://doi.org/10.1136/gut.2007.125542 (2007). 
68. Carrion, M. et al. VIP impairs acquisition of the macrophage proinflammatory polarization profile. $J$ Leukoc Biol.100, 1385-1393 https://doi.org/10.1189/jlb.3A0116-032RR (2016).

69. Dorsam, S. T. et al. Identification of the early VIP-regulated transcriptome and its associated, interactome in resting and activated murine CD4 T cells. Mol Immunol.47, 1181-1194 https://doi.org/10.1016/j.molimm.2010.01.003 (2010).

70. Belkaid, Y. \& Hand, T. W. Role of the microbiota in immunity and inflammation. Cell.157, 121-141 https://doi.org/10.1016/j.cell.2014.03.011 (2014).

71. Lara-Marquez, M., O'Dorisio, M., O'Dorisio, T., Shah, M. \& Karacay, B. Selective gene expression and activation-dependent regulation of vasoactive intestinal peptide receptor type 1 and type 2 in human T cells. J Immunol.166, 2522-2530 https://doi.org/10.4049/jimmunol.166.4.2522 (2001).

72. Heimesaat, M. M. et al. Intestinal Microbiota Changes in Mice Lacking Pituitary Adenylate Cyclase Activating Polypeptide (PACAP) - Bifidobacteria Make the Difference. European journal of microbiology \& immunology.7, 187-199 https://doi.org/10.1556/1886.2017.00021 (2017).

73. Delgado, M., Pozo, D. \& Ganea, D. The significance of vasoactive intestinal peptide in immunomodulation. Pharmacol Rev.56, 249-290 https://doi.org/10.1124/pr.56.2.7 (2004).

74. Colwell, C. S. et al. Disrupted circadian rhythms in VIP- and PHI-deficient mice. Am J Physiol Regul Integr Comp Physiol.285, R939-949 https://doi.org/10.1152/ajpregu.00200.200300200.2003[pii] (2003).

75. Walters, W. A. et al. PrimerProspector: de novo design and taxonomic analysis of barcoded polymerase chain reaction primers. Bioinformatics.27, 1159-1161 https://doi.org/10.1093/bioinformatics/btr087 (2011).

76. Caporaso, J. G. et al. Global patterns of $16 \mathrm{~S}$ rRNA diversity at a depth of millions of sequences per sample. Proc. Natl. Acad. Sci. U.S.A.108 Suppl 1, 4516-4522, doi:10.1073/pnas.1000080107 (2011).

77. Loy, A., Maixner, F., Wagner, M. \& Horn, M. probeBase-an online resource for rRNA-targeted oligonucleotide probes: new features 2007. Nucleic Acids Res.35, D800-804 https://doi.org/10.1093/nar/gkl856 (2007).

78. Magoc, T. \& Salzberg, S. L. FLASH: fast length adjustment of short reads to improve genome assemblies. Bioinformatics.27, 2957-2963 https://doi.org/10.1093/bioinformatics/btr507 (2011).

79. Martin, M. Cutadapt removes adapter sequences from high-throughput sequencing reads. EMBnet.journal.17, 10-12 https://doi.org/10.14806/ej.17.1.200 (2011).

80. Edgar, R. C. Search and clustering orders of magnitude faster than BLAST. Bioinformatics.26, 24602461 https://doi.org/10.1093/bioinformatics/btq461 (2010).

81. et al. Using QIIME to analyze 16 S rRNA gene sequences from microbial communities. Current protocols in bioinformatics / editoral board, Andreas D. Baxevanis ... et al.JChap. 10, Unit 1017 , doi:10.1002/0471250953.bi1007s36 (2011).

82. Edgar, R. C. UPARSE: highly accurate OTU sequences from microbial amplicon reads. Nature methods.10, 996-998 https://doi.org/10.1038/nmeth.2604 (2013). 
83. Altschul, S. F. et al. Gapped BLAST and PSI-BLAST: a new generation of protein database search programs. Nucleic Acids Res.25, 3389-3402 (1997).

84. Pruesse, E. et al. SILVA: a comprehensive online resource for quality checked and aligned ribosomal RNA sequence data compatible with ARB. Nucleic Acids Res.35, 7188-7196 https://doi.org/10.1093/nar/gkm864 (2007).

85. Xia, J., Psychogios, N., Young, N. \& Wishart, D. S. MetaboAnalyst: a web server for metabolomic data analysis and interpretation. Nucleic Acids Res.37, W652-660 https://doi.org/10.1093/nar/gkp356 (2009).

86. Hammer, O., Harper, D. A. T. \& PAST Paleontological statistics software package for education and data analysis. Palaeontologia Electronica.4, 1-9 (2011).

\section{Tables}

Due to technical limitations, table 1,2 is only available as a download in the Supplemental Files section.

\section{Figures}

Figure 1

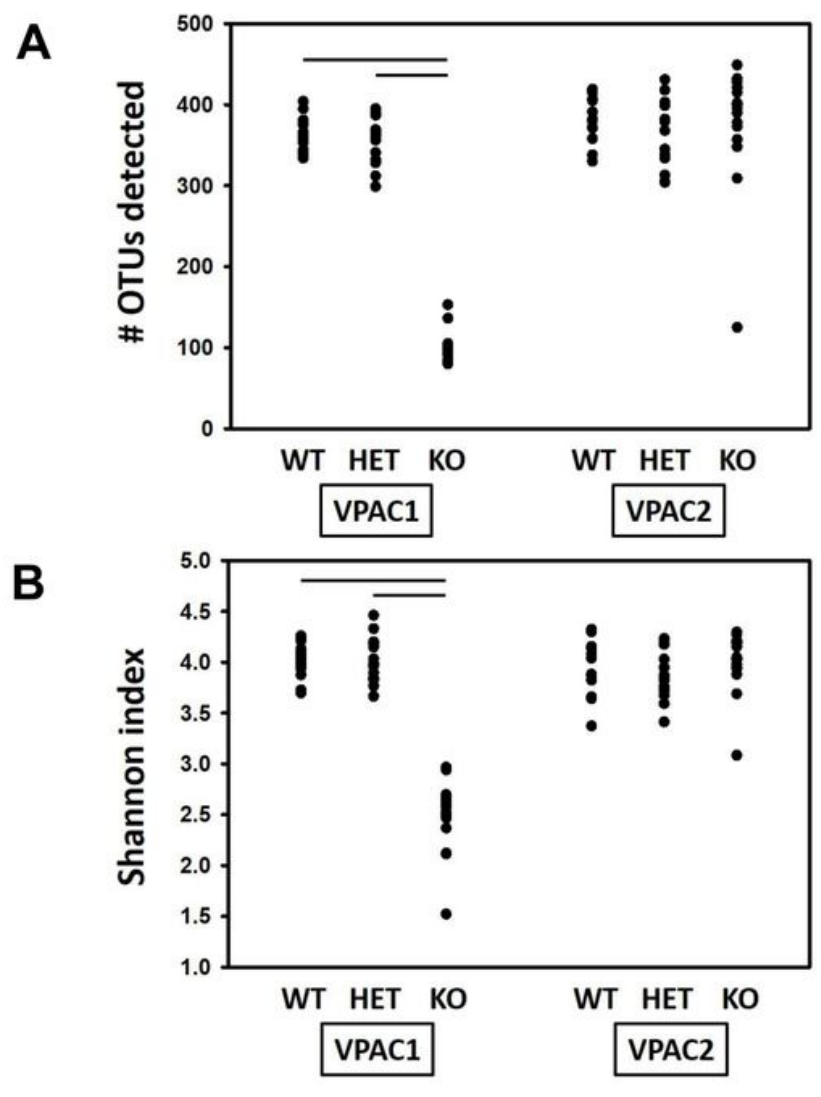

\section{Figure 1}

Biodiversity is reduced in VPAC1, but not VPAC2, KO fecal samples. Dot plots from indicated genotypes from VPAC1 and VPAC2 colonies showing (A) number of operational taxonomic units (e.g. richness) and 
(B) the Shannon index of a-diversity. Horizontal bars represent statistically significant differences in the VPAC1 colony between WT/HET and KO samples $(P \leq 0.001)$.

Figure 2A

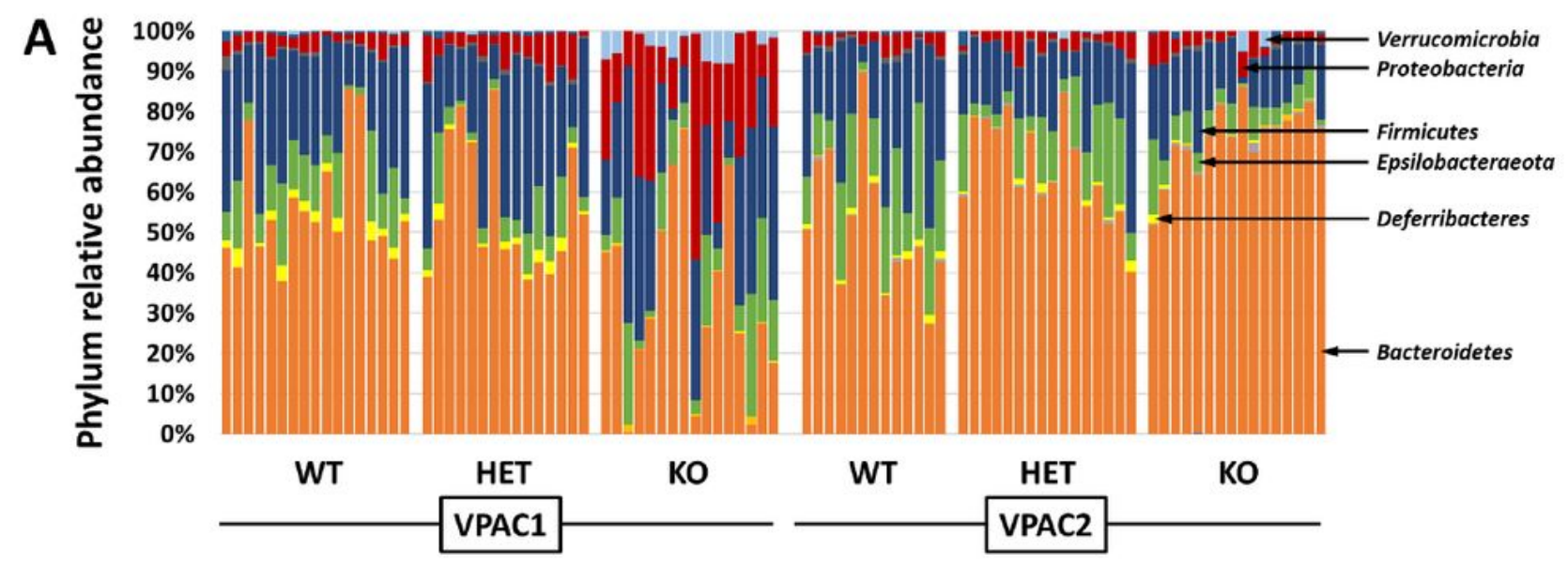

Figure 2B

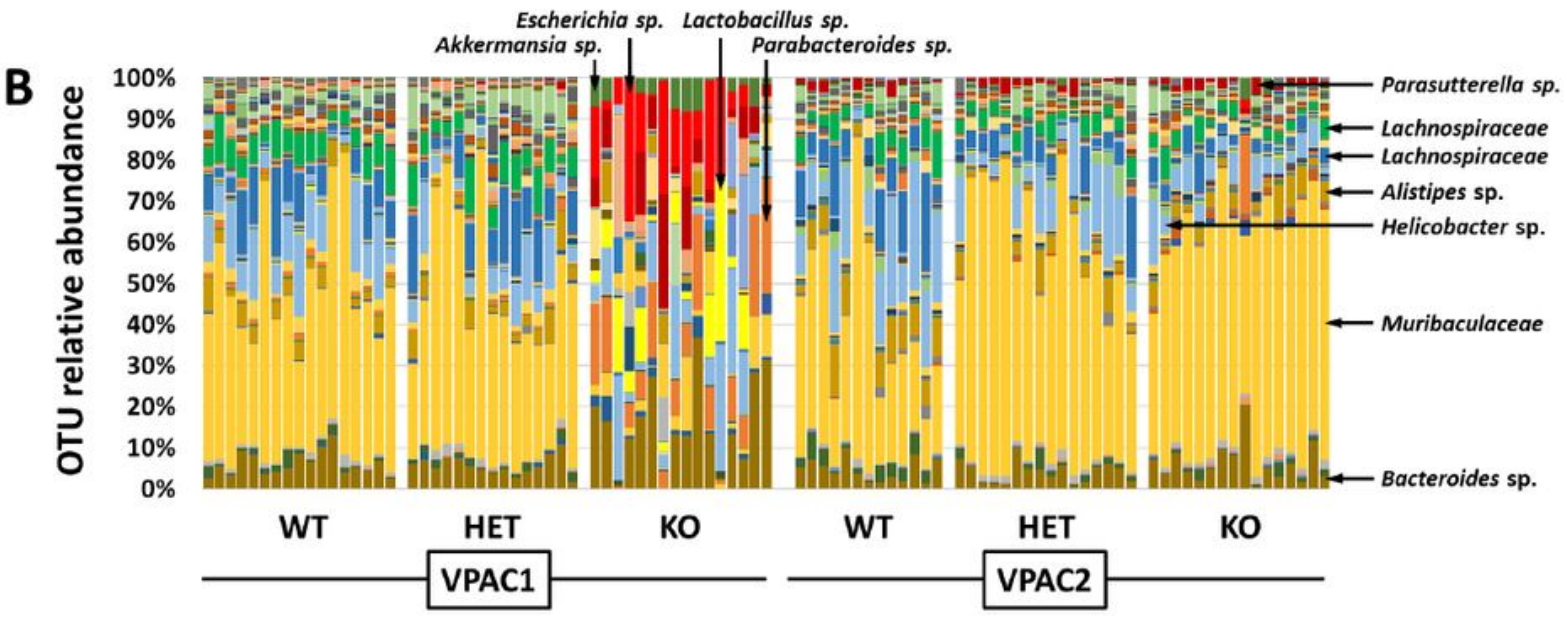

Figure 2

Net enrichment in Gram-negative bacteria from VPAC1 KO fecal samples. Vertical stacked bar charts with each color representing the relative abundance of bacterial DNA detected in each sample, annotated to 
the level of (A) phylum, and (B) operational taxonomic unit (OTU). Names of dominant OTUs are indicated with arrows.

Figure 3A-D

A

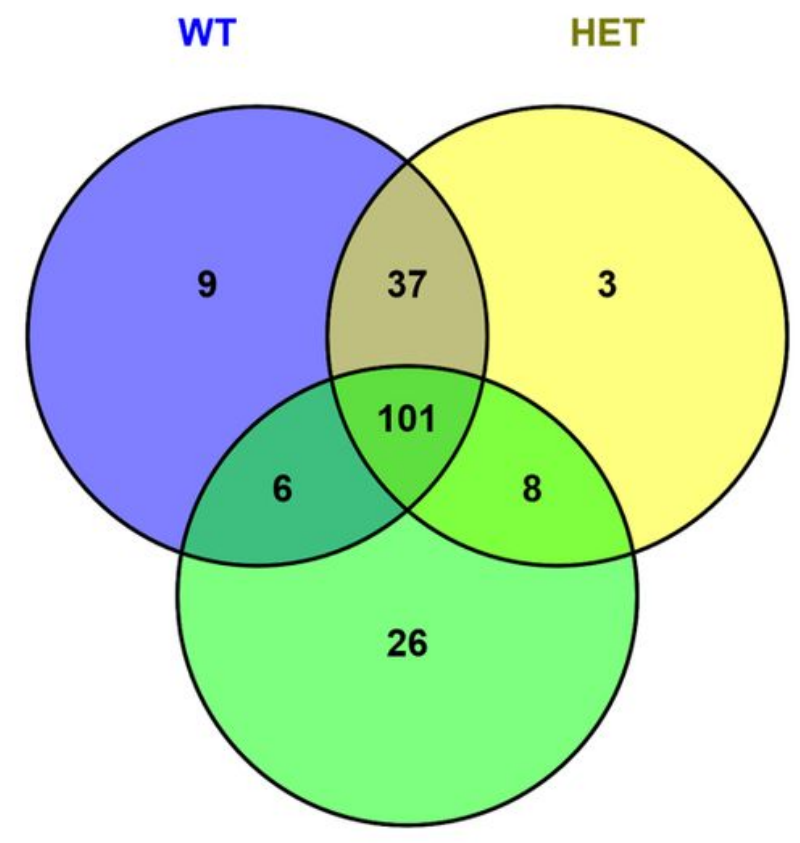

KO

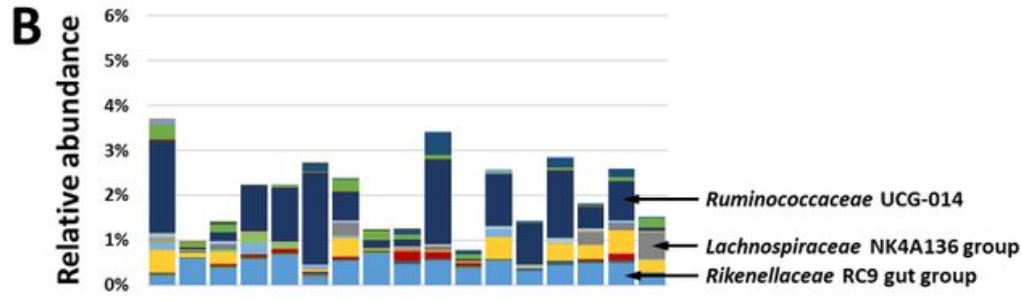

Individual WT mice

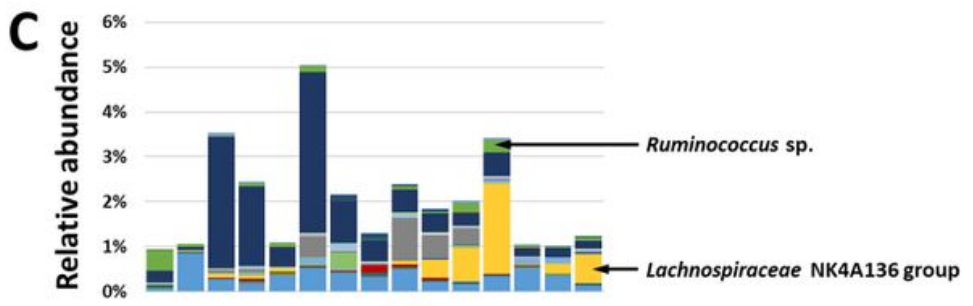

Individual HET mice

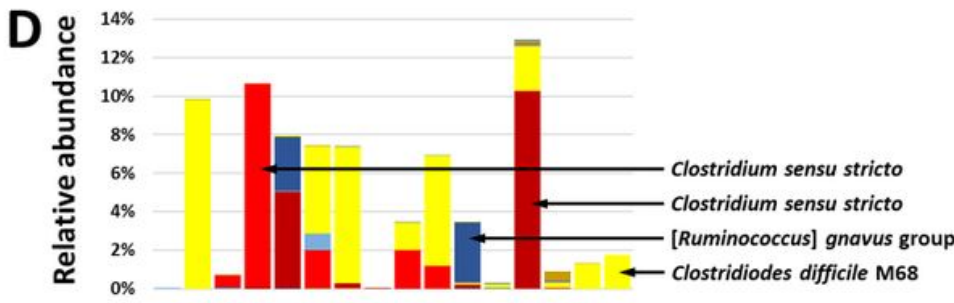

Individual KO mice

\section{Figure 3}

Reshuffling of low abundant Gram-positive bacteria from VPAC1 KO fecal samples. Venn diagram showing the overlap in community membership within the VPAC1 colony, with numbers indicating OTUs within each section of the diagram (A); the relative abundance in WT (B) and HET (C) samples of those OTUs not detected in any samples from VPAC1 KO mice; and the relative abundance in VPAC1 KO samples of OTUs not detected in any WT or HET samples (D). 

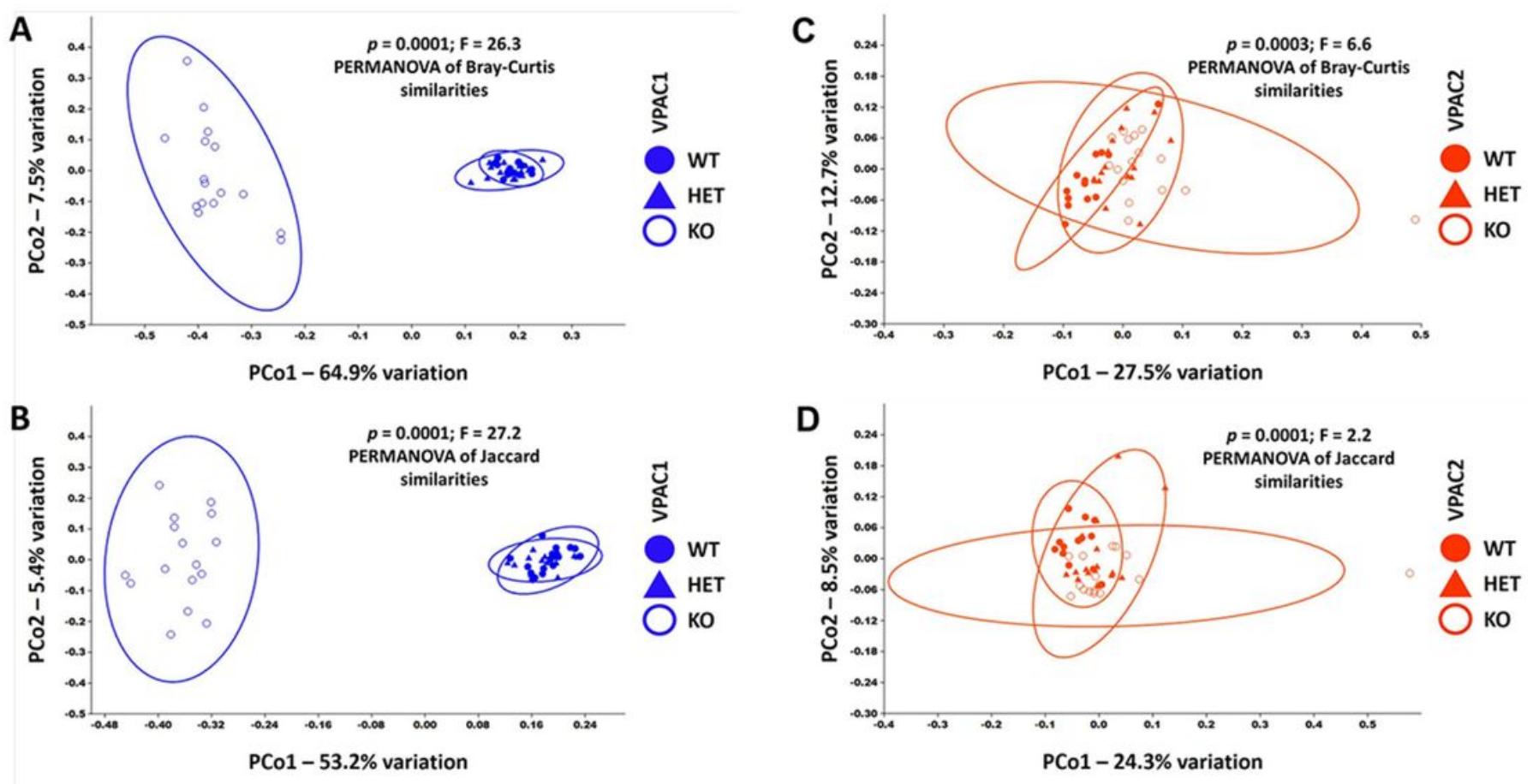

\section{Figure 4}

Statically significant clustering differences within VPAC1 and VPAC2 colonies. Principal coordinate analysis using Bray-Curtis ( $A$ and $C$ ) and Jaccard ( $B$ and $D$ ) distances to compare genotype-dependent similarities and differences within the indicated colonies denoted in legend. $\mathrm{P}$ and $\mathrm{F}$ values calculated by their respective PERMANOVA methods are indicated. 
A

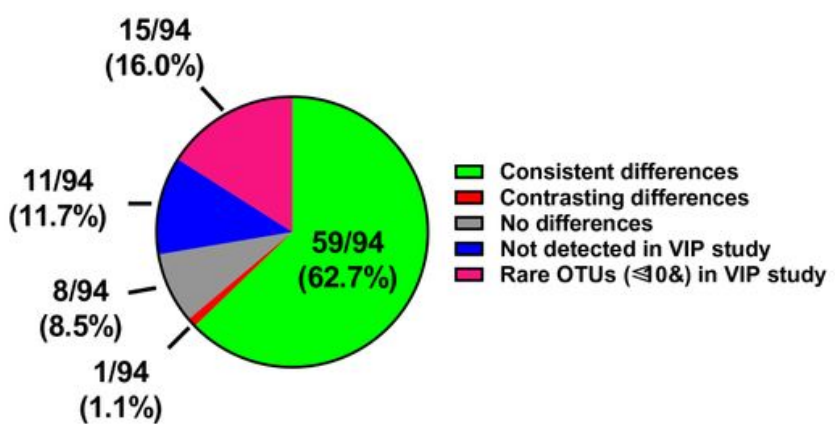

B

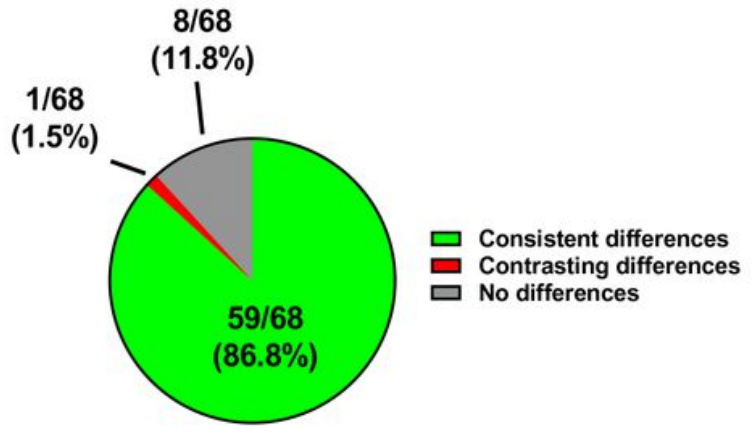

\section{Figure 5}

Consistent taxonomic changes between VIP and VPAC1 colonies. Pie chart showing the proportion of those significant VPAC1 genotype-dependent differences described in Table 2 for 94 OTUs (A) and 68 OTUs (B) also reported in the GM of VIP-deficient mice compared to WT littermates.

\section{Supplementary Files}

This is a list of supplementary files associated with this preprint. Click to download.

- Table1.pdf

- Table2.pdf

- SupplementaryFigure1.pdf

- SupplementaryFigure2AB.pdf 